\title{
COVID-19: No longer status quo for the surgeon
}

\author{
J John, K Kesner \\ Department of Urology, Frere Hospital and Walter Sisulu University, South Africa
}

Corresponding author, email: jeffveenajohn@gmail.com

Contagious viruses have plagued humanity since time immemorial. The Bubonic Plague (Black Death) and small pox in the $14^{\text {th }}$ Century, Spanish Flu in 1918, and more recently HIV/AIDS, Ebola, Middle East Respiratory Syndrome (MERS) and Severe Acute Respiratory Syndrome (SARS) are those ingrained in our memory banks. The latest culprit, a close relation to both SARS and MERS, however, threatens to disrupt and collapse the very best of health systems.

On the $31^{\text {st }}$ of December 2019, the World Health Organization (WHO) China Country Office was informed of cases of pneumonia of unknown aetiology detected in Wuhan City in the Hubei Province of China. Twelve days later, China publicly shared the genetic sequence of the 2019 novel Coronavirus and the WHO subsequently named the causative virus SARS Coronavirus 2 (SARS-CoV-2) and the resulting pathology Coronavirus Disease 2019 (COVID-19). At the time of writing, there have been 4733349 reported cases worldwide, with 313384 people (6.52\%) deaths. ${ }^{1}$ On the African continent, cases are steadily increasing. Fortunately, we are not seeing the exponential daily growth in confirmed cases, nor in mortality, witnessed in the United States and Western Europe. South Africa (SA) has the highest burden of disease in Africa. Of the 83365 confirmed cases on the continent, 14355 (17.2\%) are in South Africa. Within the country, there is significant variation in the distribution of cases. The Western Cape (WC), Gauteng and Eastern Cape (EC) account for 58.5\%, 15.8\% and 12.6\% of the national cases respectively. Alarmingly WC and EC combined currently account for $91 \%$ of all new cases over the most recently reported 24 -hour period. ${ }^{2}$

Most hospitals in SA already have strict measures in place to limit the spread of the virus. These include screening of all patients and visitors entering the hospital grounds, limiting outpatient numbers to urgent cases, increasing the intervals of outpatient visits, insisting on the wearing of masks, encouraging social distancing in the waiting rooms and frequent handwashing. In addition, public health measures to trace, test, isolate and appropriately quarantine those infected are in place to try and get definitive control of the epidemic.

Surgeons have an important role to play in helping our colleagues on the front-line curb this unprecedented pandemic. ASSA and FoSAS have advised that all nonurgent cases be cancelled or postponed and where possible, non-surgical treatment modalities should be considered.
This allows surgeons, anaesthetists and other theatre staff to be redeployed to other areas of need, protects patients from in-hospital viral transmission, eases the burden on bed numbers and reduces the utilisation of personal protective equipment (PPE).

When surgery cannot be deferred, patients should be screened on admission for possible COVID-19 and anyone found to be a person under investigation (PUI) for COVID-19 should have urgent definitive testing. If the patient's condition permits, surgery should be deferred until definitive results are released. In an emergency setting, in the absence of a recent definitive negative result, all patients must be treated as PUIs for COVID-19 and all staff need to take necessary precautions with adequate PPE.

South Africa has not been spared from the global shortage of PPE. Prices of surgical masks have increased six-fold, N95 respirators have more than trebled, and gowns cost twice as much. For these reasons, stocks are often sold to the highest bidder. ${ }^{3}$ While every effort must be taken to protect all healthcare workers, it is imperative that PPE is not misused. Guidelines set out by national bodies, including the National Institute of Communicable Disease (NICD), need to be strictly adhered to. ${ }^{4}$

Elective theatre lists have been suspended in most units in SA and surgeons from all disciplines need to professionally negotiate amongst themselves for limited theatre space. A discussion on COVID-19 should form part of the informed consent for any surgical procedure and the risks of exposing patients to possible SARS-CoV-2 infection during the admission period must be weighed against the risks of protracted treatment delay. Relatives should be advised of the need to limit their numbers visiting the hospital, even during curtailed visiting hours. The attending doctor must ensure the family is kept abreast of the patient's condition telephonically to prevent undue stress to the family and patient.

Surgery should be undertaken in a dedicated COVID-19 theatre. Anaesthesia should be performed by suitably experienced anaesthetists using established protocols that mitigate the ability of SARS-CoV-2 particles to survive up to threehours in aerosols. ${ }^{5}$ In this edition, a consensus statement by the South African Society of Endoscopic Surgeons EXCO, addresses the safety of minimally invasive surgery during the COVID-19 pandemic. ${ }^{6}$ Postoperatively patients should remain in theatre until fully recovered prior to transfer to a COVID-19-dedicated ward. 
COVID-19 will likely be with us for the remainder of 2020 and probably well into 2021. In the absence of a vaccine or any known medical cure, we are left with many unanswered questions. How long can we continue to postpone elective surgery? The British Journal of Surgery released a report from CovidSurg Collaborative, a research network of 5000 surgeons from 120 countries. It has projected that based on a 12-week period of peak distribution to hospital services due to COVID-19, 28.4 million elective surgeries will be cancelled or postponed this year. In SA, each additional week of disruption to hospital services results in an additional 12 000 surgeries being cancelled. ${ }^{7}$ How will we manage to clear the backlog? Will there be an injection of both financial and human resources to ramp up elective surgeries? It has been estimated that a financial injection more than $£ 2$ billion (R45 billion) would be necessary to clear the backlog in the United Kingdom. ${ }^{7}$ How long can diagnostic procedures be delayed, before the disease progresses to a more advanced state? In this issue FoSAS is providing guidance as to how the return to normal should be managed. ${ }^{8}$ How much will this pandemic affect the front-line healthcare workers given their higher risk of infection? The risk of death and their mental well-being are major concerns which will need to be addressed continually. The training and progression of undergraduate and postgraduate students has thrown distance and e-learning into a melting pot. The opinion piece in this edition addresses several aspects including surgical research that COVID-19 has imposed on surgical registrars.

Finally, and most importantly, how much will we learn from this pandemic, to prepare us for the next? COVID-19 isn't the first threatening disease that's surged around the world - nor will it be the last. After each pandemic, we need to be better prepared than the last. Let's hope we do better next time round.

To concur with Honourable President Cyril Ramaphosa at his COVID-19 address - "A very different South Africa and world awaits us. The greatest test will be our willingness to embrace change. Let the surgical community rise to meet this challenge."
So, let us rise...

\section{REFERENCES}

1. Worldometer. Coronavirus cases [Internet]. 2020 [cited 2020 May 17]. Available from: https://www.worldometers.info/ coronavirus/coronavirus-cases/\#daily-cases.

2. Department of Health, Republic of South Africa. Update on Covid-19 (16 May 2020) - SA Corona Virus Online Portal [Internet]. 2020 [cited 2020 May 17]. Available from: https:// sacoronavirus.co.za/2020/05/16/update-on-covid-19-16thmay-2020/.

3. Mahase E. Covid-19: Hoarding and misuse of protective gear is jeopardising the response, WHO warns. BMJ 2020;368:m869.

4. National Institute of Communicable Diseases. COVID-19 guidelines [Internet]. 2020 [cited 2020 May 17]. Available from: https://www.nicd.ac.za/diseases-a-z-index/covid-19/ covid-19-guidelines/.

5. Van Doremalen N, Bushmaker T, Morris DH, et al. Aerosol and surface stability of SARS-CoV-2 as compared with SARS-CoV-1. N Engl J Med. 2020; 382:1564-7.

6. Nel D, Oodit R, Bougard H, Panieri E on behalf of SASES EXCO. Consensus statement regarding minimally invasive surgery during the COVID-19 pandemic. Southern African Journal of Surgery. 2020;58(2):59-60. https://doi. org/10.17159/2078-5151/2020/v58n2a3330.

7. CovidSurg Collaborative, Nepogodiev D, Bhangu A. Elective surgery cancellations due to the COVID-19 pandemic: global predictive modelling to inform surgical recovery plans. $\mathrm{Br} \mathrm{J}$ Surg [Internet] 2020. Available from: http://www.ncbi.nlm. nih.gov/pubmed/32395848.

8. Van Waart J, Matley PJ, Brand M on behalf of FoSAS. Federation of Surgeons of South Africa consensus document for the resumption of elective surgery after level 5 COVID-19 "lockdown" period in South Africa. Southern African Journal of Surgery. 2020;58(2):61-63. https://doi.org/ 10.17159/2078$5151 / 2020 / v 58$ n2a3335. 